\title{
Inversion of a Thunderstorm Cloud Charging Model Based on a 3D Atmospheric Electric Field
}

\author{
Wei Xu, Cancan Zhang, Xinyuan Ji and Hongyan Xing * \\ Jiangsu Key Laboratory of Meteorological Observation and Information Processing, Nanjing University of \\ Information Science and Technology, Nanjing 210044, China; xw@nuist.edu.cn (W.X.); \\ 20172210148@nuist.edu.cn (C.Z.); jxyuan@nuist.edu.cn (X.J.) \\ * Correspondence: xinghy@nuist.edu.cn
}

Received: 30 October 2018; Accepted: 12 December 2018; Published: 16 December 2018

\begin{abstract}
The measurement of the atmospheric electric field is of great significance for the study of thunderstorm cloud charge models. Traditional electric field meters can only measure the vertical component of the atmospheric electric field, and thus it is difficult to invert the structure of the thunderstorm cloud. A three-dimensional atmospheric electric field meter was developed to simultaneously measure the horizontal and vertical components of the atmospheric electric field in this paper. The effective measurement linearly relates the measured electric field to the induced voltage, and the nonlinear equations of the three-dimensional atmospheric electric field and the thunderstorm cloud-charging model parameters were derived. The particle swarm optimization algorithm (PSO) and the three-dimensional atmospheric electric field were used to invert the thunderstorm clouds. Experimental observations of the three-dimensional electric field in a cloud during a thunderstorm were analyzed. Combined with the typical charged structure model, parameters such as the charge and relative distance of the thunderstorm cloud were determined. The results showed that the value of the inversion fitness function reached 0.7288 , and the charge structure was even. The measurement of the three-dimensional atmospheric electric field provides a new means of observation for the study of atmospheric electricity.
\end{abstract}

Keywords: three-dimensional atmospheric electric field; charged structure; particle swarm optimization algorithm; dipole

\section{Introduction}

The charging model of thunderstorm clouds refers to the use of parameters to describe the characteristics of the charge distribution inside a cloud. Atmospheric electric field is an important parameter of atmospheric physics. The average intensity of the atmospheric electric field near the ground is about $130 \mathrm{~V} / \mathrm{m}$ on fine days. The atmospheric electric field will increase significantly during the formation of thunderstorm clouds. The inversion of the thundercloud charge model from ground electric field measurement data has important theoretical value for monitoring lightning and early warning research.

The most direct way to study the charge structure of thunderstorm clouds is to use sounding balloons to detect the thunderstorm clouds and obtain the electric field distribution characteristics of the detection equipment on the flight path. Simpson, Marshall, and others proposed the model of the tripole charge structure of a thunderstorm cloud by using sounding balloon observations [1]. Due to the short flying time of the sounding balloon, the measured electric field is the instantaneous charge structure of the thunderstorm cloud, so it is difficult to realize continuous monitoring. In recent years, scholars have used numerical simulation methods combined with the micro-dynamic factors of cloud physics to simulate the charge structure characteristics during the development of thunderstorm 
clouds [2-6]. However, most of these methods are simulations, and there is no physical quantity that combines actual observations.

Atmospheric electric field meters play an important role in the detection of thunderstorm clouds. Studies [7-10] have looked at the improvement of atmospheric electric field meters and the method of electric field-based lightning warning. However, current techniques can only measure the vertical electric field component, ignoring the horizontal electric field caused by the charge of the thunderstorm cloud. The literature [11-13] has proposed the classical thunderstorm cloud charge model and used the atmospheric electric field data to study the structural parameters of the model. The data used are still derived from an atmospheric electric field that can only measure the vertical component [14]. As the horizontal component is neglected, it is necessary to invert the data from the joint observations of atmospheric electric field meters at different locations. However, the atmospheric electric field is greatly affected by the building and surface conditions at the installation site, leading to a difference in each atmospheric electric field measurement. The consistency is not high, and the quality of the data participating in the inversion is poor [15].

This paper attempted to develop a three-dimensional atmospheric electric field meter capable of simultaneously measuring horizontal and vertical components, and more comprehensively establish a functional relationship between the thunderstorm cloud charge structure and the electric field of the observation point. Combined with the particle swarm algorithm, the measurement data inversion using a single three-dimensional electric field meter was used. The structural parameters of thunderstorm clouds provides a new technical means for the study of the charge structure of thunderstorm clouds.

In this paper, the electric field generated by thunderstorm clouds on the ground was observed by using the developed three-dimensional electric field meter, and compared with the radar observation data. The experimental results showed that the trajectory of the thunderstorm cloud could be analyzed by using the three-dimensional electric field data. At the same time, using the particle swarm algorithm (PSO) and the three-dimensional atmospheric electric field data, the thunderstorm cloud-charged structure of the observation point (Nanjing) can exhibit the classic dipole. These conclusions are not achievable with conventional single-dimensional electric field meters.

In Section 2, we analyzed the measurement and calibration principle of the three-dimensional electric field meter we developed. A method for inversion of thunderstorm cloud charge structure using ground three-dimensional electric field data was proposed. In Section 3, the observation experiment of a thunderstorm cloud was carried out by using the developed three-dimensional electric field meter, and the experimental results were analyzed. In Section 4, the advantages of the three-dimensional atmospheric electric field meter in the lightning warning and the inversion of the charged structure parameters were compared with the traditional single-dimensional atmospheric electric field meter. In the last part, the application prospect of 3D electric field meter was prospected, and its limitations were pointed out.

\section{Materials and Methods}

\subsection{Principles of Measurement and Calibration}

The physical and structural details of the three-dimensional electric field sensor we developed are shown in Figure 1. It consisted mainly of a rotating shield; an induction electrode perpendicular to the radial $X$, radial $Y$, and axial $Z$ directions; a photoelectric switch; a synchronous blade; and a motor. The sensing electrodes $X$ and $Y$ were composed of two opposite rectangular metal sheets distributed on the side of the circular body. The sensing electrode $Z$ was a metal disk that was equally divided into eight sectors. The three sensing electrodes were perpendicular to each other. When the motor drove the shield to rotate at a constant speed, the electrodes $X, Y, Z$ and the atmospheric electric field were periodically shielded and contacted, and an induced current was generated on the electrodes. The magnitude of the induced current was proportional to the atmospheric electric field, and the small blades were continuously driven by the motor. The shield rotated synchronously, so that the 
photoelectric switch generated a square wave signal of the same frequency and the same phase, which was convenient for phase sensitive detection of the induced voltage.
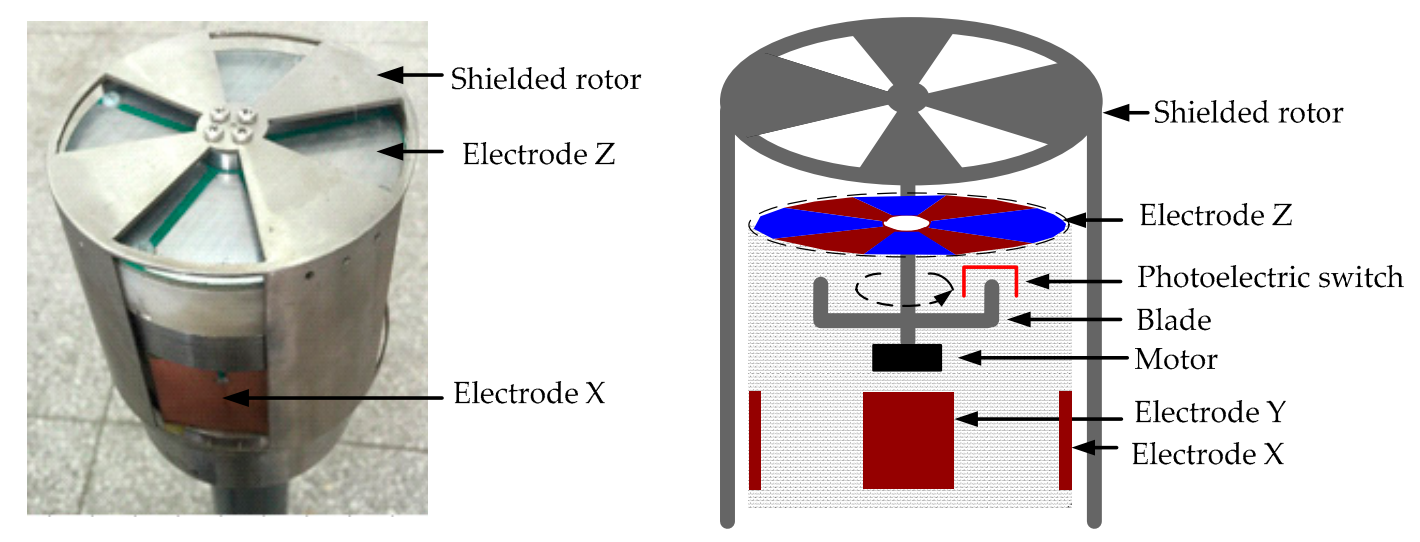

Figure 1. A photograph (left) and schematic (right) of the three-dimensional atmospheric electric field sensor.

The traditional atmospheric electric field meter only had an electrode $Z$ and a corresponding rotating shield on the bottom of the cylinder. Because the shield rotated periodically on the bottom surface, the $Z$ electrode was periodically exposed to the vertical electric field, resulting in induced current. This kind of electric field meter could only measure the vertical component of the atmospheric electric field and was suitable for measuring the atmospheric electric field on sunny days, because the horizontal component of the atmospheric electric field was very weak on sunny days. The two pairs of parallel electrodes were added to the side of the cylinder and the shield was extended along the side. When the shield was rotated, the electrodes on both sides and the bottom of the cylinder were periodically exposed to the space electric field, and the induced current was generated at the same time. It was suitable for detecting horizontal and vertical electric fields in thunderstorms.

The electrodes in all three directions were composed of two symmetrical metal pieces, which could generate differential induced currents and suppress common mode noise. The $X$ and $Y$ electrodes were each composed of two rectangular metal sheets distributed at opposite positions on the sides of the cylinder. The width of each metal piece (chord) corresponded to an arc length of $1 / 8$ of the entire circumference. The $Z$ electrode was composed of two metal sheets, $A$ and $B$ (blue fans $A$ and brown fans $B$ in Figure 1). The shielding cover rotation frequency was $f$; the atmospheric dielectric constant was $\varepsilon_{0}$; the electric field intensity in the three directions were $E_{x}, E_{y}$, and $E_{z}$; and the induced charges were $q_{x}(t), q_{y}(t)$, and $q_{z}(t)$. The area of a single metal piece of the $X$ and $Y$ electrodes was $S$. The outer radius of the $Z$ electrode disk was $r_{2}$, and the inner radius was $r_{1}$. With the rotation of the shield, the three sensing electrodes were gradually exposed from time 0 , and the electrodes were completely exposed at time $\frac{T}{2}$. During the time $\frac{T}{2}-T$, the three electrodes were gradually blocked by the shield. Then, the induced currents $i_{x(y)}(t)$ and $i_{z}(t)$, generated by one of the metal pieces of the $X$ $(Y)$ electrode and by the $A$ portion of the $Z$ electrode, respectively, were:

$$
\begin{gathered}
i_{z}(t)=\frac{d q_{z}(t)}{d t}=\left\{\begin{array}{c}
-4 \pi \varepsilon_{0} E_{z} f\left(r_{2}{ }^{2}-r_{1}{ }^{2}\right) t \in\left(0, \frac{T}{2}\right) \\
4 \pi \varepsilon_{0} E_{z} f\left(r_{2}{ }^{2}-r_{1}{ }^{2}\right) t \in\left(\frac{T}{2}, T\right)
\end{array}\right. \\
i_{x(y)}(t)=\frac{d q_{x(y)}(t)}{d t}=\left\{\begin{array}{c}
-\sqrt{2} \pi \varepsilon E_{X(Y)} f S \cos \left(\frac{\pi}{4}-2 \pi f t\right) t \in\left(0, \frac{T}{2}\right) \\
\sqrt{2} \pi \varepsilon E_{X(Y)} f S \cos \left(\frac{\pi}{4}-2 \pi f t\right) t \in\left(\frac{T}{2}, T\right)
\end{array}\right.
\end{gathered}
$$

It could be seen that when the rotational frequency $f$ of the motor was a constant value, the induced current generated by the $X, Y$ and $Z$ electrodes was linear with the external electric field. 
Furthermore, the periods of the induced currents in the three directions were identical. The signal was calculated by measuring the induced voltage to determine the electric field intensity.

The structure of the sensor causes distortion of the electric field near the sensing electrode, so the output voltage of the sensing electrode in each direction is not only related to the electric field in the direction perpendicular to the electrode, but is also weakly affected by the electric field in other directions. For the three-dimensional electric fields $E_{x}, E_{y}$, and $E_{z}$, the measured induced voltage amplitudes of the three electrodes were $U_{x}, U_{y}$, and $U_{z}$, respectively. When the electric field intensity in all three directions was 0 , the induced voltages generated by the three electrodes were $U_{x 0}, U_{y 0}$, and $U_{z 0}$, respectively. Thus:

$$
\left|\begin{array}{c}
U_{X}-U_{X 0} \\
U_{Y}-U_{Y 0} \\
U_{Z}-U_{Z 0}
\end{array}\right|=\left|\begin{array}{lll}
a_{0} & b_{0} & c_{0} \\
a_{1} & b_{1} & c_{1} \\
a_{2} & b_{2} & c_{2}
\end{array}\right|\left|\begin{array}{c}
E_{X} \\
E_{Y} \\
E_{Z}
\end{array}\right|
$$

where the matrix of $a_{i}, b_{i}$, and $c_{i}$ represented calibration coefficients. To determine the calibration values, a DC voltage was applied to a parallel plate capacitor to generate standard electric fields, which were perpendicular to the $X, Y$, and $Z$ electrodes, and the actual output voltage of the sensor was measured. Based on the measured voltage and voltage values, the calibration factor was calculated using Equation (3).

\subsection{The Relationship between a Three-Dimensional Atmospheric Electric Field and Thunderstorm Cloud Charge}

The three-dimensional atmospheric electric field meter installed in the field is shown in Figure 2a. To solve the relationship between the atmospheric electric field at the observation point and the thunderstorm cloud charge, an observation model (Figure $2 b$ ) was established. The $X-Y$ plane was mounted on the ground; $A(x, y, z)$ was an arbitrary observation point; $B\left(x_{0}, y_{0}, z_{0}\right)$ was the equivalent charge point of thunderstorm cloud; and the charge of the thunderstorm cloud was $q$. The horizontal azimuth of the thunderstorm cloud $B$ relative to the observation point $A$ was $\alpha$, the elevation angle was $\gamma$, and $A^{\prime}$ and $B^{\prime}$ were projections of the observation station $A$ and the thunderstorm cloud $B$ on the $X-Y$ plane, respectively.

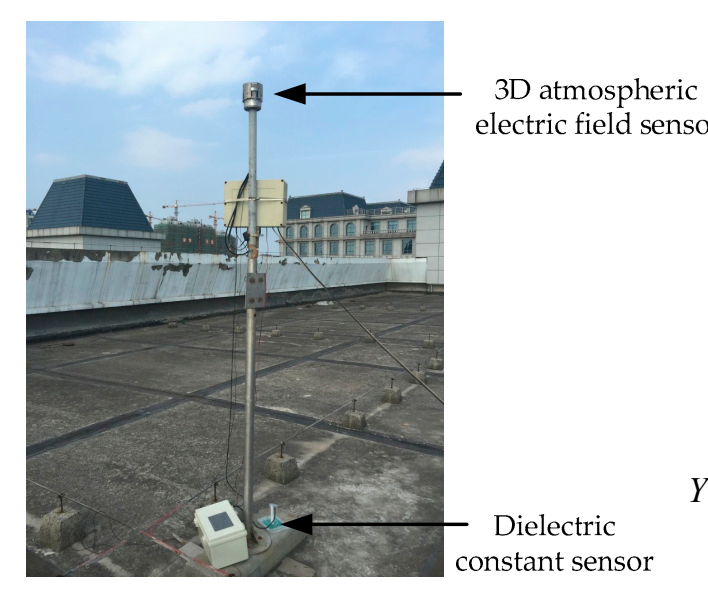

(a)

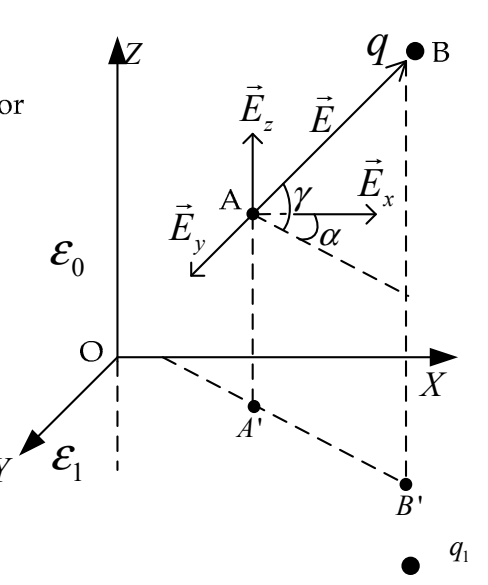

(b)

Figure 2. Observation experiment and model of the three-dimensional atmospheric electric field meter. (a) Installation of a three-dimensional atmospheric electric field meter; (b) observation model.

According to the geometric relationship of Figure $2 b$, the horizontal azimuth angle $\alpha$ and the elevation angle $\gamma$ of the thunderstorm cloud satisfied:

$$
\alpha=\arctan \left|\frac{y-y_{0}}{x-x_{0}}\right|
$$




$$
\gamma=\arctan \frac{\left|z_{0}-z\right|}{\sqrt{\left(x-x_{0}\right)^{2}+\left(y-y_{0}\right)^{2}}}
$$

The dielectric constants of region 0 above the surface $X-Y$ and region 1 inside the earth were $\varepsilon_{0}$ and $\varepsilon_{1}$, respectively. The electric field of region 0 was generated by the thunderstorm cloud charge $q$ and the induced charge of the surface. The induced charge was equivalent to the image charge $q_{1}$. The distance between the observation point $A$ and charge $q$ and its image charge $q_{1}$ were $R_{0}$ and $R_{1}$, respectively. The electric field of region 1 was generated by charge $q$ and the image charge $q_{0}$ of region 0 , and the distance of the image charge $q_{0}$ from the observation point $A$ was also $R_{0}$. The potentials at any point above the surface $(z>0)$ and inside the earth $(z<0)$ are shown in Equations (6) and (7), respectively:

$$
\begin{gathered}
\varphi_{0}=\frac{1}{4 \pi \varepsilon_{0}}\left(\frac{q}{R_{0}}+\frac{q_{1}}{R_{1}}\right)=\frac{1}{4 \pi \varepsilon_{0}}\left(\frac{q}{\sqrt{\left(x-x_{0}\right)^{2}+\left(y-y_{0}\right)^{2}+\left(z-z_{0}\right)^{2}}}+\frac{q_{1}}{\sqrt{\left(x-x_{0}\right)^{2}+\left(y-y_{0}\right)^{2}+\left(z+z_{0}\right)^{2}}}\right) \\
\varphi_{1}=\frac{1}{4 \pi \varepsilon_{1}}\left(\frac{q_{0}+q}{R_{0}}\right)=\frac{1}{4 \pi \varepsilon_{1}}\left(\frac{q_{0}+q}{\sqrt{\left(x-x_{0}\right)^{2}+\left(y-y_{0}\right)^{2}+\left(z-z_{0}\right)^{2}}}\right)
\end{gathered}
$$

The boundary conditions at $z=0$ are shown in Equations (8) and (9):

$$
\begin{aligned}
\varphi_{0} & =\varphi_{1} \\
\varepsilon_{0} \frac{\partial \varphi_{0}}{\partial z} & =\varepsilon_{1} \frac{\partial \varphi_{1}}{\partial z}
\end{aligned}
$$

Finally, the image charges can be obtained from Equations (6)-(9):

$$
\begin{gathered}
q_{0}=\frac{\varepsilon_{1}-\varepsilon_{0}}{\varepsilon_{1}+\varepsilon_{0}} q \\
q_{1}=-\frac{\varepsilon_{1}-\varepsilon_{0}}{\varepsilon_{1}+\varepsilon_{0}} q
\end{gathered}
$$

By substituting Equations (10) and (11) into Equation (6), one can obtain the potential $\varphi_{0}$ at the observation point $A$, as shown in Equation (12):

$$
\varphi_{0}=\frac{1}{4 \pi \varepsilon_{0}}\left(\frac{q}{R_{0}}+\frac{q_{1}}{R_{1}}\right)=\frac{1}{4 \pi \varepsilon_{0}}\left(\frac{q}{\sqrt{\left(x-x_{0}\right)^{2}+\left(y-y_{0}\right)^{2}+\left(z-z_{0}\right)^{2}}}-\frac{\varepsilon_{1}-\varepsilon_{0}}{\varepsilon_{1}+\varepsilon_{0}} \frac{q}{\sqrt{\left(x-x_{0}\right)^{2}+\left(y-y_{0}\right)^{2}+\left(z+z_{0}\right)^{2}}}\right)
$$

The potential scalar function was used to derive the partial derivatives of $x, y$, and $z$. The value $z=0$ was used to obtain the electric field component produced by the charged $q$ of thunderstorm cloud $B\left(x_{0}, y_{0}, z_{0}\right)$ at the ground observation point; that is, the installation position $A(x, y, z)$ of the three-dimensional electric field meter:

$$
\begin{aligned}
& \overrightarrow{E_{x}}=\left[\frac{q\left(x-x_{0}\right)}{2 \pi\left(\varepsilon_{0}+\varepsilon_{1}\right)}\left[\left(x-x_{0}\right)^{2}+\left(y-y_{0}\right)^{2}+z_{0}^{2}\right]^{-\frac{3}{2}}\right] \overrightarrow{e_{x}} \\
& \overrightarrow{E_{y}}=\left[\frac{q\left(y-y_{0}\right)}{2 \pi\left(\varepsilon_{0}+\varepsilon_{1}\right)}\left[\left(x-x_{0}\right)^{2}+\left(y-y_{0}\right)^{2}+z_{0}^{2}\right]^{-\frac{3}{2}}\right] \overrightarrow{e_{y}} \\
& \overrightarrow{E_{z}}=\left[-\frac{q z_{0}}{2 \pi \varepsilon_{0}} \frac{\varepsilon_{1}}{\varepsilon_{0}+\varepsilon_{1}}\left[\left(x-x_{0}\right)^{2}+\left(y-y_{0}\right)^{2}+z_{0}^{2}\right]^{-\frac{3}{2}}\right] \overrightarrow{e_{z}}
\end{aligned}
$$

where $\overrightarrow{e_{x}}, \overrightarrow{e_{y}}$ and $\overrightarrow{e_{z}}$ were the unit vectors of $\overrightarrow{E_{x}}, \overrightarrow{E_{y}}$ and $\overrightarrow{E_{z}}$ respectively, and the direction is as shown in Figure 1 , which was in the same direction as $\vec{E}_{x}, \vec{E}_{y}$ and $\vec{E}_{z}$. 
Based on Equations (4) and (5) for the elevation and azimuth angles of thunderstorm clouds and on Equations (13)-(15) of the three-dimensional electric field components, the horizontal and elevation angles of the charge center $B$ of thunderstorm clouds relative to the ground observation stations could be calculated from the three-dimensional atmospheric electric field component of the observation point $(z=0)$ :

$$
\begin{gathered}
\alpha=\arctan \left|\frac{y-y_{0}}{x-x_{0}}\right|=\arctan \left|\frac{E_{y}}{E_{x}}\right| \\
\gamma=\arctan \frac{\left|z_{0}\right|}{\sqrt{\left(x-x_{0}\right)^{2}+\left(y-y_{0}\right)^{2}}}=\arctan \frac{\varepsilon_{0}\left|E_{z}\right|}{\varepsilon_{1} \sqrt{E_{x}^{2}+E_{y}^{2}}}
\end{gathered}
$$

Combined with the polarity of the electric field, the new three-dimensional electric field meter determined the horizontal and elevation angles of the thunderstorm cloud charge relative to the observation point by measuring the dielectric constant and electric field component of the installed surface in real time, so as to realize the thunderstorm cloud location in complex surface environments and different weather conditions.

\subsection{Nonlinear Inversion Method for Thunderstorm Cloud Charge Parameters}

The charge region in the thunderstorm cloud is mainly related to the structural characteristics of the temperature layer in which it is located [16]. The charge region in the cloud is evenly distributed in the horizontal direction, and is only related to the vertical height. It was assumed that the thunderstorm cloud was divided into several equally thick layers, and the charge of each layer was regarded as a point charge, as shown in Figure 3. The ground three-dimensional electric field value was the result of the interaction of multiple charge regions in the thunderstorm cloud. The ground three-dimensional electric field value was determined as in Equations (18)-(20):

$$
\begin{gathered}
E_{x}=\sum_{k=1}^{n}\left[\frac{Q_{k}\left(x-x_{0}\right)}{2 \pi\left(\varepsilon_{0}+\varepsilon_{1}\right)}\left[\left(x-x_{0}\right)^{2}+\left(y-y_{0}\right)^{2}+\left(z_{0}+(k-1) h+\frac{h}{2}\right)^{2}\right]^{-\frac{3}{2}}\right] \\
E_{y}=\sum_{k=1}^{n}\left[\frac{Q_{k}\left(y-y_{0}\right)}{2 \pi\left(\varepsilon_{0}+\varepsilon_{1}\right)}\left[\left(x-x_{0}\right)^{2}+\left(y-y_{0}\right)^{2}+\left(z_{0}+(k-1) h+\frac{h}{2}\right)^{2}\right]^{-\frac{3}{2}}\right] \\
E_{z}=\sum_{k=1}^{n}\left[-\frac{Q_{k}\left(z_{0}+(k-1) h+\frac{h}{2}\right)}{2 \pi\left(\varepsilon_{0}+\varepsilon_{1}\right)}\left[\left(x-x_{0}\right)^{2}+\left(y-y_{0}\right)^{2}+\left(z_{0}+(k-1) h+\frac{h}{2}\right)^{2}\right]^{-\frac{3}{2}}\right]
\end{gathered}
$$

where $(x, y, 0)$ and $\left(x_{0}, y_{0}, z_{0}\right)$ were the coordinates of the ground three-dimensional electric field meter and the thunderstorm cloud, respectively. $h$ was the thickness of the thunderstorm cloud charge, $k$ was the index that indicated the charge zone layers, $Q_{k}$ was the charge of the $k$ layer charge zone, and $n$ was the total number of charge layers.

Based on the functional relationship between the ground three-dimensional atmospheric electric field and the charge of the thunderstorm cloud (Equations (18)-(20)), combined with the classical thunderstorm cloud charge model, the parameters of the charge structure of the thunderstorm cloud-including the charge quantity $Q_{k}$ of each layer and the relative distance between the thunderstorm cloud and observation point $\left|x-x_{0}\right|,\left|y-y_{0}\right|, z_{0}$ could be inverted by using the ground three-dimensional electric field data.

Generally, the Newton iteration method is a common method for calculating non-linear equations. Each iteration direction is along the direction of the current point function value descending. However, the selection of the initial value of iteration is very important. The least square method is usually used to determine the initial value of iteration. The combination of the two algorithms can transform the non-linear problem into a linear one. Due to the existence of $x-x_{0}$ and $y-y_{0}$ in Equations (18) 
and (19) and their high dimensions, it is difficult to convert to the form of linear $\mathrm{AX}=\mathrm{B}$, and the initial values of Newton's method cannot be solved by the least square method. Therefore, other non-linear inversion algorithms were considered.

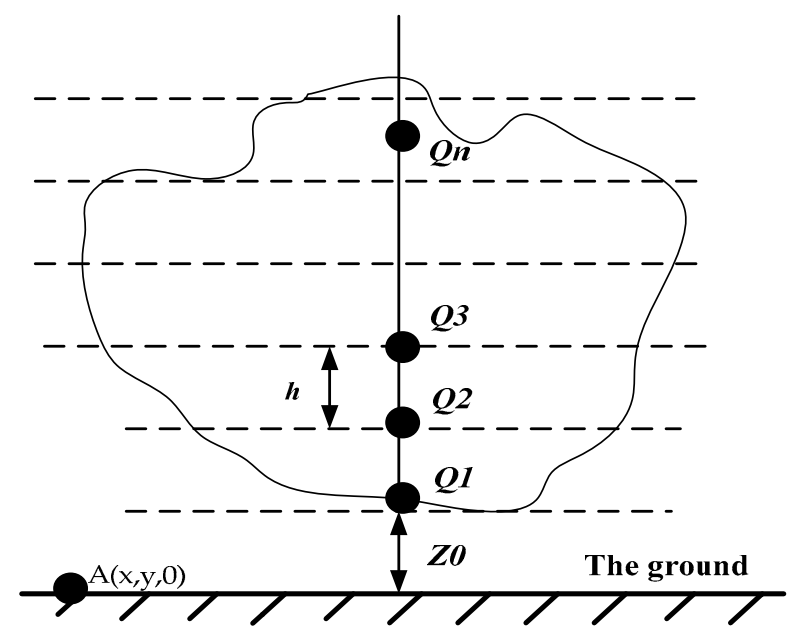

Figure 3. Schematic diagram of the thunderstorm cloud charge differentiation layer.

Genetic algorithm and particle swarm optimization are commonly used in geophysical non-linear inversion. In the process of solving nonlinear inversion, the genetic algorithm does not have a memory function. The solution obtained will change with the charge structure of thunderstorm cloud in the inversion process, and easily fall into local optimum, so it is difficult to obtain truly satisfied structural parameters. In the process of solving parameters of the three-dimensional electric field $\left\{E_{x}, E_{y}, E_{z}\right\}$, the convergence speed of genetic algorithm is slow. Particle swarm optimization (PSO) has memory function and can well preserve all the charge structure parameters with the optimal solution probability. It only updates the internal velocity of the particle and has fast convergence speed $[17,18]$. The problem of slow convergence of the three-dimensional inversion process can be well solved. Therefore, the particle swarm optimization (PSO) algorithm is suitable to inverse the charge structure of thunderstorm cloud.

The $D$-dimensional search space of the function randomly generates solid particles. These particles determine their movement direction by comparing the current best position and the historical best position of themselves or other particles. They approach the target by a constant updating cycle of their own speed and position, and finally find the optimal solution of the objective function as a whole [19]. The steps of using particle swarm optimization to retrieve the structural parameters of the thunderstorm cloud were as follows:

(1) Initialization of the charge structure parameters In order to avoid blind searching of structural parameters in the inversion process, the velocity and position of structural parameters need to be initialized. Combined with the classical charged structural parameters of thunderstorm clouds, the inversion parameters are limited to a certain range $\left[-V_{\max }, V_{\max }\right]$ and $\left[-X_{\max }, X_{\max }\right]$, the initial velocity of charge parameter $Q_{k}$ should be $[-5,5]$, the relative distance between the thunderstorm clouds and three-dimensional atmospheric electric field meter should be assumed. The initial speed of the parameter $\left(\left|x-x_{0}\right|,\left|y-y_{0}\right|, z_{0}\right)$ is $[-100,100]$. The initial location of the charge parameter is $[-30,30]$, and the initial location of the relative distance is $[-10,000,10,000]$.

(2) Fitness function of the three-dimensional electric field The difference between the measured value $E$ of the ground electric field and the inverted ground electric field value $E^{*}$ is defined as a fitness function. When value $Y$ of the fitness function approaches zero, the optimal solution of the parameters can be obtained:

$$
E=\left\{E_{x}, E_{y}, E_{z}\right\}
$$




$$
\begin{gathered}
E^{*}=\left\{E^{*}{ }_{x}, E^{*}{ }_{y}, E^{*}{ }_{z}\right\} \\
Y\left(Q_{1}, Q_{2}, \ldots, Q_{k}, x-x_{0}, y-y_{0}, z_{0}\right)=\left\{E_{x}-E_{x}{ }^{*}, E_{y}-E_{y}{ }^{*}, E_{z}-E_{z}{ }^{*}\right\}
\end{gathered}
$$

(3) Find the initial extremum of the inversion parameter set Assuming the population size $i$ of the charged structure parameter is 5 and the dimension of search space $D$ is the number of inversion variables, and assuming the thunderstorm cloud is a classical three-layer charged structure, then $D=9$. That is, the inversion parameter set is $\left\{Q_{1}, Q_{2}, \ldots, Q_{k}, Q_{1}{ }^{\prime}, Q_{2}{ }^{\prime} \ldots, Q_{k}{ }^{\prime},\left|x-x_{0}\right|,\left|y-y_{0}\right|, z_{0}\right\}$. If it is a two-layer charged structure, then $D=5$, that is, the set of inversion parameters is $\left\{Q_{1}, Q_{2}, \ldots, Q_{k},\left|x-x_{0}\right|,\left|y-y_{0}\right|, z_{0}\right\}$. The initial extremum $P_{\text {best }}$ of each parameter of the charged structure is the position of the individual when the initial fitness is optimal, and the global initial extremum $G_{b e s t}$ is the position with the best fitness searched in the $i$ parameter sets. Let the initial velocity of the $i$ structural parameters be $V_{i}=\left[V_{i 1}, V_{i 2}, \ldots, V_{i D}\right]^{T}$, the individual initial extremum $P_{i}$ is $P_{i}=\left[P_{i 1}, P_{i 2}, \ldots, P_{i D}\right]^{T}$, and the population initial global extremum $G_{i}$ is $G_{i}=\left[G_{i 1}, G_{i 2}, \ldots, G_{i D}\right]^{T}$.

(4) Update of inversion parameters The velocity and position of the structural parameters in the $k+1$ iteration are obtained by updating the individual extremum $P_{b e s t}$ and the global extremum $G_{\text {best }}$ of the $k$ th iteration. Each time the position is updated, the fitness value $Y$ is calculated once, and the position update and speed update are shown in Equations (24) and (25):

$$
\begin{gathered}
X_{i d}^{k+1}=X_{i d}^{k}+V_{i d}^{k+1} \\
V_{i d}^{k+1}=w V_{i d}^{k}+c_{1} r_{1}\left(P_{i d}^{k}-X_{i d}^{k}\right)+c_{2} r_{2}\left(P_{g d}^{k}-X_{g d}^{k}\right)
\end{gathered}
$$

where $V_{i d}^{k}$ and $V_{i d}^{k+1}$ are the convergence speeds of the $k$ th and $k+1$ th structural parameters, respectively, and $X_{i d}^{k}$ and $X_{i d}^{k+1}$ are the structural parameter values of the $k$ and $k+1$ times, respectively. $c_{1}$ and $c_{2}$ are acceleration factors that determine the ability of particles to search for global optimal values and individual optimal values. $d$ is the number of inverse evolutions, $w$ is the weight coefficient, and $P_{i d}^{k}$ is the $i$ th particle at the $k$ th iteration. $P_{g d}^{k}$ is the global extremum of the $i$ th structural parameter set at the $k$ th iteration.

\section{Experimental Results and Analysis}

\subsection{Three-Dimensional Atmospheric Electric Field and Radar Observation Experiments}

More than one year of observation experiments were carried out using our three-dimensional atmospheric electric field meter [20]. Figure 4 is a three-dimensional electric field curve for the period from 14:35 p.m. to 17:15 p.m. on 9 October 2018. It can be seen from the figure that the change of the ground electric field caused by the thunderstorm cloud can be divided into four stages. In the first stage (14:35 p.m.-15:25 p.m.), the atmospheric electric field $E_{z}$ on the ground appeared to fluctuate continuously and the amplitude began to increase. The charge carried by the thunderstorm cloud entered the detection range of the three-dimensional atmospheric electric field meter, but was also far away. In the second stage (15:30 p.m.-15:50 p.m.), due to the discharge phenomenon, the atmospheric electric field $E_{z}$ oscillated greatly, the polarity was reversed multiple times, and the charging and discharging process was obvious. The thunderstorm cloud is located directly above the observation point. In the third stage (15:50 p.m.-16:25 p.m.), the atmospheric electric field $E_{z}$ gradually weakens, and the horizontal electric field and electric field amplitude reach a peak multiple times. It was judged that the thunderstorm cloud was located in the east direction of the observation point and the distance was relatively close. In the fourth stage, after 16:25 p.m., $E_{x}, E_{y}$, and $E_{z}$ gradually decreased and tended to be stable, and the thunderstorm cloud gradually moved away from the detection range. 


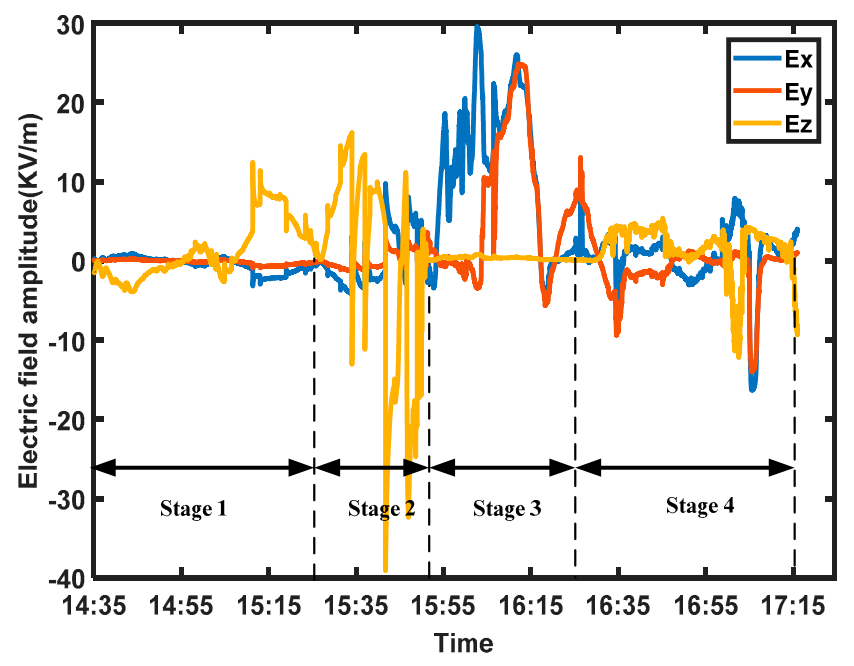

Figure 4. Three-dimensional atmospheric electric field curve.

At the same place, the radar map of the same location from 15:06 p.m. to 16:12 p.m. are shown in Figure 5. The central point of the circle was the observation point of the three-dimensional atmospheric electric field meter $A$. It can be clearly seen that at 15:06 p.m., the thunderstorm cloud was far away from the observation point, and the amplitudes of $E_{x}, E_{y}$ and $E_{z}$ were smaller. At 15:30 p.m., the thunderstorm cloud approaches the observation point $A$ gradually, and the $E_{z}$ component increases. At 15:48 p.m., the thunderstorm cloud is located directly above the three-dimensional atmospheric electric field meter, and the $E_{z}$ component measured is strong. At this time, thunderstorm occurrence is predicted. At 16:12 p.m., the thunderstorm cloud over the region moves southeast, still within the detection range, and $E_{x}$ and $E_{y}$ increase gradually. The movement of thunderstorm clouds in this period is consistent with that measured by three-dimensional atmospheric electric field meter.
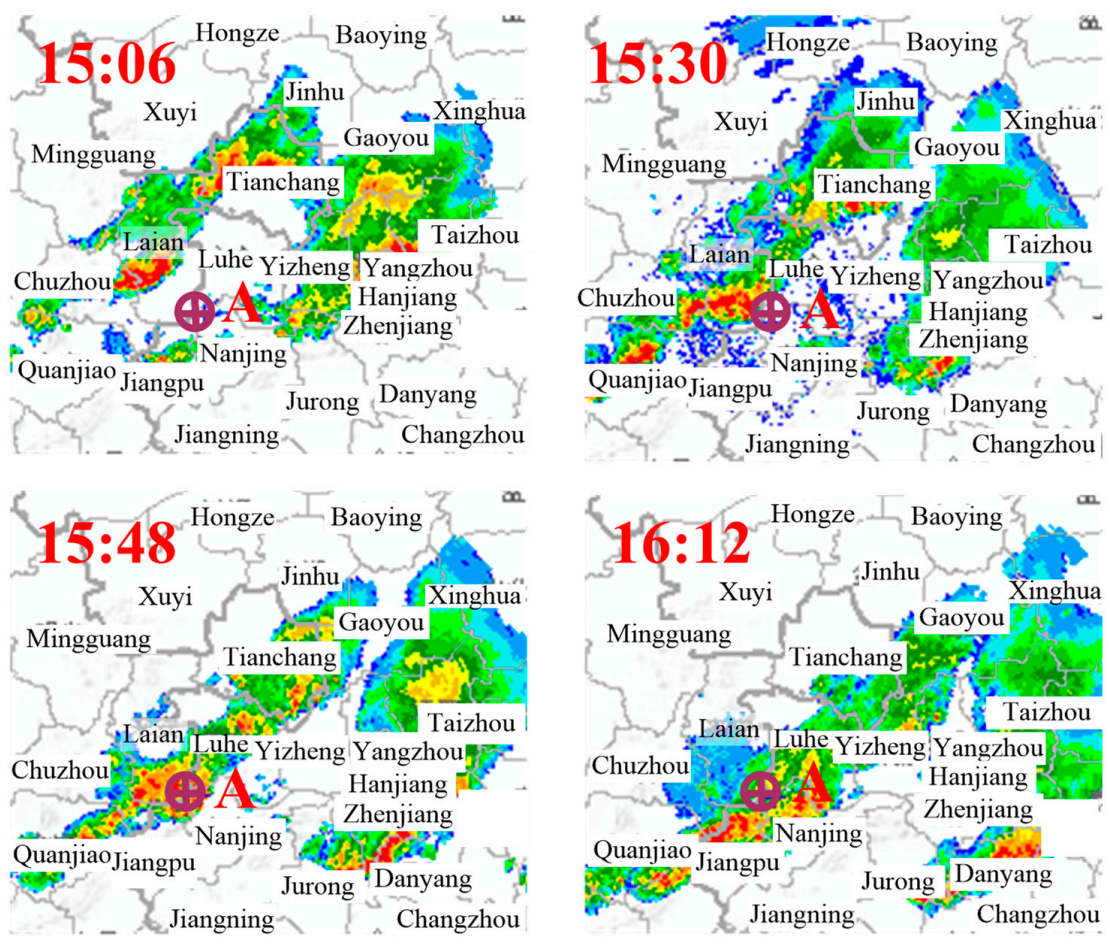

Figure 5. Radar map. 


\subsection{Inversion of the Charge Model}

According to the observation data of radar and atmospheric electric field meters, the classical three-layer charging model and the structural inversion of the two-layer charging model were respectively performed on the thunderstorm cloud at a certain time point of a second stage thunderstorm. The sampling interval of the three-dimensional atmospheric electric field meter was $1 \mathrm{~s}$, and the measured data of the three-dimensional electric field at two adjacent sampling moments were $7.299 \mathrm{kV} / \mathrm{m}, 2.219 \mathrm{kV} / \mathrm{m},-29.560 \mathrm{kV} / \mathrm{m}$ and $7.222 \mathrm{kV} / \mathrm{m}, 2.183 \mathrm{kV} / \mathrm{m},-29.207 \mathrm{kV} / \mathrm{m}$. Due to the extremely short time, the observation point was considered. The relative distance $\left(\left|x-x_{0}\right|,\left|y-y_{0}\right|\right.$, $z_{0}$ ) from the thunderstorm cloud was approximately constant. A set of nonlinear equations between the three-dimensional electric field value and each charge structure parameter was established as per Equations (26)-(31):

$$
\begin{aligned}
& E_{x 1}=\frac{Q_{1}\left(x-x_{0}\right)}{2 \pi\left(\varepsilon_{0}+\varepsilon_{1}\right)}\left[\left(x-x_{0}\right)^{2}+\left(y-y_{0}\right)^{2}+\left(z_{0}+500\right)^{2}\right]^{-\frac{3}{2}}+\frac{Q_{2}\left(x-x_{0}\right)}{2 \pi\left(\varepsilon_{0}+\varepsilon_{1}\right)}\left[\left(x-x_{0}\right)^{2}+\left(y-y_{0}\right)^{2}\right. \\
& \left.+\left(z_{0}+1500\right)^{2}\right]^{-\frac{3}{2}}+\frac{Q_{3}\left(x-x_{0}\right)}{2 \pi\left(\varepsilon_{0}+\varepsilon_{1}\right)}\left[\left(x-x_{0}\right)^{2}+\left(y-y_{0}\right)^{2}+\left(z_{0}+2500\right)^{2}\right]^{-\frac{3}{2}} \\
& E_{y 1}=\frac{Q_{1}\left(y-y_{0}\right)}{2 \pi\left(\varepsilon_{0}+\varepsilon_{1}\right)}\left[\left(x-x_{0}\right)^{2}+\left(y-y_{0}\right)^{2}+\left(z_{0}+500\right)^{2}\right]^{-\frac{3}{2}}+\frac{Q_{2}\left(y-y_{0}\right)}{2 \pi\left(\varepsilon_{0}+\varepsilon_{1}\right)}\left[\left(x-x_{0}\right)^{2}+\left(y-y_{0}\right)^{2}\right. \\
& \left.+\left(z_{0}+1500\right)^{2}\right]^{-\frac{3}{2}}+\frac{Q_{3}\left(y-y_{0}\right)}{2 \pi\left(\varepsilon_{0}+\varepsilon_{1}\right)}\left[\left(x-x_{0}\right)^{2}+\left(y-y_{0}\right)^{2}+\left(z_{0}+2500\right)^{2}\right]^{-\frac{3}{2}} \\
& E_{z 1}=\frac{-Q_{1} \varepsilon_{1}\left(z_{0}+500\right)}{2 \pi \varepsilon_{0}\left(\varepsilon_{0}+\varepsilon_{1}\right)}\left[\left(x-x_{0}\right)^{2}+\left(y-y_{0}\right)^{2}+\left(z_{0}+500\right)^{2}\right]^{-\frac{3}{2}}+\frac{-Q_{2} \varepsilon_{1}\left(z_{0}+1500\right)}{2 \pi \varepsilon_{0}\left(\varepsilon_{0}+\varepsilon_{1}\right)}\left[\left(x-x_{0}\right)^{2}+\left(y-y_{0}\right)^{2}\right. \\
& \left.+\left(z_{0}+1500\right)^{2}\right]^{-\frac{3}{2}}+\frac{-Q_{3} \varepsilon_{1}\left(z_{0}+2500\right)}{2 \pi \varepsilon_{0}\left(\varepsilon_{0}+\varepsilon_{1}\right)}\left[\left(x-x_{0}\right)^{2}+\left(y-y_{0}\right)^{2}+\left(z_{0}+2500\right)^{2}\right]^{-\frac{3}{2}} \\
& E_{x 1}{ }^{\prime}=\frac{Q_{1}{ }^{\prime}\left(x-x_{0}\right)}{2 \pi\left(\varepsilon_{0}+\varepsilon_{1}\right)}\left[\left(x-x_{0}\right)^{2}+\left(y-y_{0}\right)^{2}+\left(z_{0}+500\right)^{2}\right]^{-\frac{3}{2}}+\frac{Q_{2}{ }^{\prime}\left(x-x_{0}\right)}{2 \pi\left(\varepsilon_{0}+\varepsilon_{1}\right)}\left[\left(x-x_{0}\right)^{2}+\left(y-y_{0}\right)^{2}\right. \\
& \left.+\left(z_{0}+1500\right)^{2}\right]^{-\frac{3}{2}}+\frac{Q{ }^{\prime}\left(x-x_{0}\right)}{2 \pi\left(\varepsilon_{0}+\varepsilon_{1}\right)}\left[\left(x-x_{0}\right)^{2}+\left(y-y_{0}\right)^{2}+\left(z_{0}+2500\right)^{2}\right]^{-\frac{3}{2}} \\
& E_{y 1}{ }^{\prime}=\frac{Q_{1}{ }^{\prime}\left(y-y_{0}\right)}{2 \pi\left(\varepsilon_{0}+\varepsilon_{1}\right)}\left[\left(x-x_{0}\right)^{2}+\left(y-y_{0}\right)^{2}+\left(z_{0}+500\right)^{2}\right]^{-\frac{3}{2}}+\frac{Q_{2}{ }^{\prime}\left(y-y_{0}\right)}{2 \pi\left(\varepsilon_{0}+\varepsilon_{1}\right)}\left[\left(x-x_{0}\right)^{2}+\left(y-y_{0}\right)^{2}\right. \\
& \left.+\left(z_{0}+1500\right)^{2}\right]^{-\frac{3}{2}}+\frac{Q_{3}{ }^{\prime}\left(y-y_{0}\right)}{2 \pi\left(\varepsilon_{0}+\varepsilon_{1}\right)}\left[\left(x-x_{0}\right)^{2}+\left(y-y_{0}\right)^{2}+\left(z_{0}+2500\right)^{2}\right]^{-\frac{3}{2}} \\
& E_{z 1}{ }^{\prime}=\frac{-Q_{1} \varepsilon_{1}\left(z_{0}+500\right)}{2 \pi \varepsilon_{0}\left(\varepsilon_{0}+\varepsilon_{1}\right)}\left[\left(x-x_{0}\right)^{2}+\left(y-y_{0}\right)^{2}+\left(z_{0}+500\right)^{2}\right]^{-\frac{3}{2}}+\frac{-Q_{2}{ }^{\prime} \varepsilon_{1}\left(z_{0}+1500\right)}{2 \pi \varepsilon_{0}\left(\varepsilon_{0}+\varepsilon_{1}\right)}\left[\left(x-x_{0}\right)^{2}+\left(y-y_{0}\right)^{2}\right. \\
& \left.+\left(z_{0}+1500\right)^{2}\right]^{-\frac{3}{2}}+\frac{-Q_{3}^{\prime} \varepsilon_{1}\left(z_{0}+2500\right)}{2 \pi \varepsilon_{0}\left(\varepsilon_{0}+\varepsilon_{1}\right)}\left[\left(x-x_{0}\right)^{2}+\left(y-y_{0}\right)^{2}+\left(z_{0}+2500\right)^{2}\right]^{-\frac{3}{2}}
\end{aligned}
$$

According to the inversion method in the previous section, the parameters of the three-layer charged model of thunderstorm clouds were inverted by using the three-dimensional atmospheric electric field measurements. The convergence curve is shown in Figure 6. The inversion results are shown in Table 1 , where $Q_{1}, Q_{2}, Q_{3}, Q_{1}{ }^{\prime}, Q_{2}{ }^{\prime}$, and $Q_{3}{ }^{\prime}$ are the charges of the thunderstorm clouds, $\left|x-x_{0}\right|,\left|y-y_{0}\right|$, and $z_{0}$ are the relative distances between the observation points of the ground three-dimensional electric field meter and the thunderstorm clouds.

Table 1. Parameters of the three-layer charging model of thunderstorm clouds.

\begin{tabular}{ccccccccc}
\hline$Q_{1}$ & $Q_{2}$ & $Q_{3}$ & $Q_{1}{ }^{\prime}$ & $Q_{2}{ }^{\prime}$ & $Q_{3}{ }^{\prime}$ & $\left|x-x_{0}\right|$ & $\left|y-y_{0}\right|$ & $z_{0}$ \\
\hline $30 \mathrm{C}$ & $30 \mathrm{C}$ & $-18.38 \mathrm{C}$ & $30 \mathrm{C}$ & $30 \mathrm{C}$ & $-18.379 \mathrm{C}$ & $2.856 \mathrm{~km}$ & $863.38 \mathrm{~m}$ & $1.3115 \mathrm{~km}$ \\
\hline
\end{tabular}

It can be seen from the inversion results that the charged structure of the thunderstorm cloud had a positive charge on the uppermost layer, a negative charge on the middle layer and the bottom layer, and $\left(Q_{1}{ }^{\prime}, Q_{2}{ }^{\prime}, Q_{3}{ }^{\prime}\right)$ were approximately equal to and $\left(Q_{1}, Q_{2}, Q_{3}\right)$, respectively, which also indicated a very short time. The charge of the internal thunderstorm cloud did not change substantially, and the 
charge structure was an even-polar structure with a negative polarity, which was consistent with the classical dipole structure.

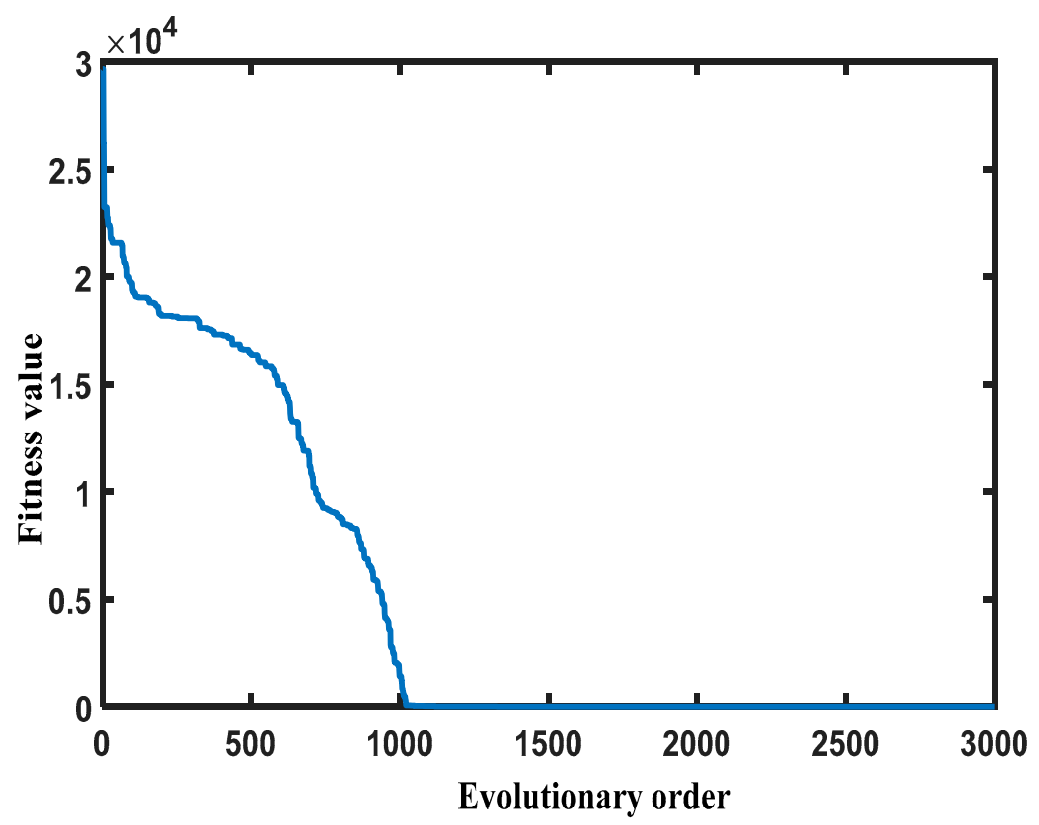

Figure 6. The convergence curve of the three-layer charge model.

\subsection{Comparative Analysis of Electric Field Data}

According to the lightning activity in Nanjing and surrounding areas, Nanjing Meteorological Bureau has established a monitoring network covering the whole city by using the traditional atmospheric electric field meter, as shown in Figure 7. Point A in the figure is the three-dimensional electric field meter (AEFM), which is located at Nanjing University of Information Science and Technology $\left(118^{\circ} 43^{\prime} 30.00^{\prime \prime}\right.$ E, $32^{\circ} 11^{\prime} 56.40^{\prime \prime}$ N). Pukou, Liuhe, Nanjing meteorological Bureau, and Kunlun are the observation stations of several conventional atmospheric electric field meters that are closest to point $\mathrm{A}$. The positive direction of $\mathrm{X}$ is the east direction, and the positive direction of $\mathrm{Y}$ is the north direction.

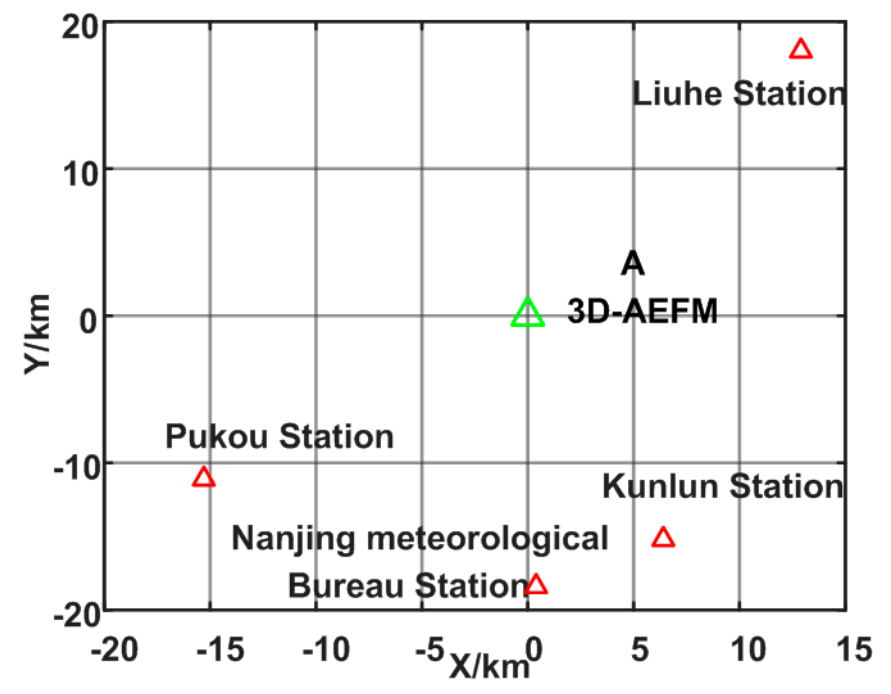

Figure 7. Monitoring network of the atmospheric electric field meter in Nanjing.

The distance between each station and the three-dimensional electric field meter is known in Figure 7. So the vertical electric field components of each station can be calculated based on the 
structural parameters of thunderstorm cloud retrieved in Table 1. The vertical electric field values obtained by the three-dimensional electric field meter were compared with the official measurements as shown in Table 2 .

Table 2. Comparisons between official data and 3D AEFM (the three-dimensional electric field meter) measurements.

\begin{tabular}{cccc}
\hline Station & Distance $(\mathrm{Km})$ & Official Data $(\mathrm{KV} / \mathrm{m})$ & 3D AEFM $(\mathrm{KV} / \mathrm{m})$ \\
\hline Pukou & 18.91 & -1.6 & -1.583 \\
Nanjing Meteorological & 18.40 & -1.4 & -1.367 \\
Bureau & 16.49 & -1.9 & -1.931 \\
Kunlun & 22.14 & -0.8 & -0.830 \\
Liuhe & &
\end{tabular}

It can be seen that the vertical electric field values at the other locations obtained by the three-dimensional electric field meter were consistent with the official measurements.

\section{Discussion and Conclusions}

The three-dimensional electric field meter we developed overcomes the shortcomings of a traditional electric field meter that can only measure the vertical electric field components at the ground. Observational experiments have confirmed that thunderstorm clouds produce horizontal electric field components that cannot be ignored near the ground. This provides more comprehensive electric field information for the tracking, warning, and charge structure of thunderstorm clouds. By establishing an observation model of the three-dimensional electric field, it was proven that the orientation information of the thunderstorm cloud can be determined by the ratio of the three-dimensional electric field values of the observation points. Therefore, the three-dimensional electric field data can be used to continuously monitor the movement trajectory of the thunderstorm cloud, which can be used as a useful supplement to radar data.

Lightning warning is an important application of atmospheric electric field meters. The traditional atmospheric electric field meter has a certain leakage alarm because it only measures the vertical atmospheric electric field and ignores the horizontal component. For example, when the three-dimensional electric field meter in the experiment measured the thunderstorm cloud in the second stage (15:50 p.m.-16:20 p.m.), the horizontal component electric fields $E_{x}$ and $E_{y}$ were obviously stronger than the vertical electric field $E_{z}$. The amplitude gave a lightning warning. However, if it was measured by a conventional electric field meter, it will be impossible to give an early warning because the electric field $E_{z}$ in the vertical direction is weak.

The amount of electric field information measured by traditional electric field meters is too small, and it is difficult to invert the structural parameters of the thunderstorm cloud. Multiple networking electric field meters must be used to calculate the positional relationship of each electric field meter and the measured vertical electric field. However, the installation environment of each electric field meter in the grid including the terrain, surrounding buildings, etc., will cause distortion of the atmospheric electric field, resulting in poor consistency of the measurement data, and it is difficult to correct. Based on incorrect electric field data, the inversion of atmospheric electric fields is difficult to carry out. The three-dimensional electric field and the charged structure parameters are nonlinear, and the particle swarm optimization algorithm is suitable for nonlinear inversion problems. However, in the process of finding the optimal solution in the inversion iteration, the position of the particle must be limited according to the typical thunderstorm cloud charge structure parameter. The inversion method assumes that the thunderstorm cloud is a multi-layer structure, and the charge center of the thunderstorm cloud is treated as a point charge. The thunderstorm cloud-charged structure of the experimental inversion observation site (Nanjing) was evenly polar, which was consistent with the results of the comprehensive analysis of the electric field and radar data in the literature [21]. 
The measurement of three-dimensional atmospheric electric field provides more comprehensive data for the study of lightning structure and lightning warning of thunderstorm clouds. In addition to ground detection, the developed three-dimensional electric field sensor can also be used in the field of high-altitude detection to measure the horizontal electric field component that cannot be ignored, which is impossible for current vertical electric field meters. It should be noted that the proposed inversion method was based on the thunderstorm cloud charge as a point charge model and was based on a classical uniform multilayer structure. Some studies believe that thunderstorm cloud-charge structures are geographically related and further work is recommended to validate this process.

Author Contributions: Conceptualization, W.X.; data curation, C.Z.; formal analysis, W.X.; investigation, X.J.; methodology, W.X.; project administration, H.X.; software, C.Z.; supervision, H.X.; writing—original draft, C.Z.; writing-review and editing, W.X.

Funding: This work is supported by the National Natural Science Foundation of China (Grant No. 41605121) and the Priority Academic Program Development of Jiangsu Higher Education Institutions.

Conflicts of Interest: The authors declare no conflict of interest.

\section{References}

1. Simpson, G.; Scrase, F.J. The Distribution of Electricity in Thunderclouds. Proc. R. Soc. Lond. 1937, 161, 309-352. [CrossRef]

2. Zhao, P.; Yin, Y.; Xiao, H.; Zhou, Y.; Liu, J. Role of Water Vapor Content in the Effects of Aerosol on the Electrification of Thunderstorms: A Numerical Study. Atmosphere 2016, 7, 137. [CrossRef]

3. Tsenova, B.D.; Barakova, D.; Mitzeva, R. Numerical study on the effect of charge separation at low cloud temperature and effective water content on thunderstorm electrification. Atmos. Res. 2017, 184, 1-14. [CrossRef]

4. Fan, X.; Zhang, Y.; Zhang, G.; Zheng, D. Lightning Characteristics and Electric Charge Structure of a Hail-Producing Thunderstorm on the Eastern Qinghai-Tibetan Plateau. Atmosphere 2018, 9, 295. [CrossRef]

5. Gao, W.; Kou, Z.; Ding, P. A Numerical Study of Hail Process Effects on Charge Structure and Lightning Flash Rate of Thunderstorms. In Proceedings of the IEEE Asia-Pacific International Symposium on Electromagnetic Compatibility, Shenzhen, China, 17-21 May 2016; pp. 594-596.

6. Galanaki, E.; Lagouvardos, K.; Kotroni, V.; Flaounas, E.; Argiriou, A. Thunderstorm climatology in the Mediterranean using cloud-to-ground lightning observations. Atmos. Res. 2018, 207, 136-144. [CrossRef]

7. Montanya, J.; Bergas, J.; Hermoso, B. Electric field measurements at ground level as a basis for lightning hazard warning. J. Electrostat. 2004, 60, 241-246. [CrossRef]

8. Ferro, M.A.D.S.; Yamasaki, J.; Pimentel, D.R.M.; Naccarato, K.P.; Saba, M.M.F. Lightning risk warnings based on atmospheric electric field measurements in Brazil. J. Aerosp. Technol. Manag. 2011, 3, 301-310. [CrossRef]

9. Zeng, Q.; Wang, Z.; Guo, F.; Feng, M.; Zhou, S.; Wang, H.; Xu, D. The application of lightning forecasting based on surface electrostatic field observations and radar data. J. Electrostat. 2013, 71, 6-13. [CrossRef]

10. Fort, A.; Mugnaini, M.; Vignoli, V.; Rocchi, S.; Perini, F.; Monari, J.; Schiaffino, M.; Fiocchi, F. Design, modeling, and test of a system for atmospheric electric field measurement. IEEE Trans. Instrum. Meas. 2011, 60, 2778-2785. [CrossRef]

11. Wilson, C.T.R. On some determinations of the sign and magnitude of electric discharges in lightning flashes. Proc. R. Soc. Lond. A 1916, A92, 555-574. [CrossRef]

12. Chen, Q.; Wei, G.; Wan, H. Quantum inversion of thunderstorm cloud charge model. J. Geophys. 2010, 53, 2237-2243.

13. Amoruso, V.; Lattarulo, F. Thundercloud pre-stroke electrostatic modeling. J. Electrostat. 2002, 56, 255-276. [CrossRef]

14. Schumann, C.; Silva, R.B.G.D.; Schulz, W. Electric fields changes produced by positives cloud-to-ground lightning flashes. J. Atmos. Sol. Terr. Phys. 2013, 92, 37-42. [CrossRef]

15. Yang, D.; Oldenburg, D.W. Electric field data in inductive source electromagnetic surveys. Geophys. Prospect. 2018, 66, 207-225. [CrossRef]

16. Jayaratne, E.R.; Saunders, C.P.R. The interaction of ice crystals with hailstones in wet growth and its possible role in thunderstorm electrification. Q. J. R. Meteorol. Soc. 2016, 142, 1809-1815. [CrossRef] 
17. Khan, S.U.; Yang, S.; Wang, L.; Liu, L. A Modified Particle Swarm Optimization Algorithm for Global Optimizations of Inverse Problems. IEEE Trans. Magn. 2016, 52, 1-4. [CrossRef]

18. Pallero, J.L.; Fernández-Muñiz, M.Z.; Cernea, A.; Álvarez-Machancoses, Ó.; Pedruelo-González, L.M.; Bonvalot, S.; Fernández-Martínez, J.L. Particle Swarm Optimization and Uncertainty assessment in Inverse Problems. Entropy 2018, 20, 96. [CrossRef]

19. Huang, Y.F.; Tan, T.H.; Chen, B.A.; Liu, S.H.; Chen, Y.F. Performance of Resource Allocation in Device-to-Device Communication Systems Based on Particle Swarm Optimization. In Proceedings of the IEEE International Conference on Systems, Man and Cybernetics, Banff, AB, Canada, 5-8 October 2017; pp. 400-404.

20. Xing, H.; He, G.; Ji, X. Analysis on Electric Field Based on Three Dimensional Atmospheric Electric Field Apparatus. J. Electr. Eng. Technol. 2018, 13, 1696-1703.

21. Wang, Z.H.; Zeng, Q.F.; Guo, F.X.; Xu, D.P.; Wang, H. A Study of the Electrostatic Field Networking in Three Isolated Thunderstorms. Appl. Mech. Mater. 2013, 239, 775-784. [CrossRef]

(c) 2018 by the authors. Licensee MDPI, Basel, Switzerland. This article is an open access article distributed under the terms and conditions of the Creative Commons Attribution (CC BY) license (http:/ / creativecommons.org/licenses/by/4.0/). 